\title{
Current opinions and practices in the treatment of spontaneous pneumothorax
}

\author{
Stewart S W Chan
}

\begin{abstract}
The approach to the initial management of spontaneous pneumothorax differs markedly from centre to centre, and it is difficult in practice to establish a standard protocol. This article reviews the concepts behind the British Thoracic Society guidelines, and reports the varying opinions and alternative practices existing currently. There is a need for more evidence-based studies to identify what is the best approach. Based on a review of relevant recent reports, the author attempts to work out an unbiased practical approach that can be used safely and that can possibly give the best overall cost effective results.

(F Accid Emerg Med 2000;17:165-169)
\end{abstract}

Keywords: spontaneous pneumothorax; management; guidelines

Several options exist for the choice of treatment for spontaneous pneumothorax. Whereas previously tube thoracostomy and hospitalisation used to be the treatment of choice for most spontaneous pneumothoraces, the past decade has seen an increasing trend towards more conservative management, by means of observation and simple aspiration with outpatient follow up. However, several problems are often encountered by the emergency physician, when opting to treat a patient with spontaneous pneumothorax conservatively. Firstly, failed aspiration with recurrent pneumothorax within 48 hours is encountered frequently. Secondly, there is uncertainty about the degree of risk involved with the conservative approach. Hence questions arise concerning the required length of time for in hospital observation, the necessity for and the best timing of repeat radiographs, and also questions concerning when intercostal tube drainage would be indicated for a patient. Since the British Thoracic Society published its guidelines for the management of spontaneous pneumothorax ${ }^{1}$ in 1993 , it seems timely at this point to look at the worldwide experience and opinions on this subject. A review of current literature has been performed, and this highlights some of the important findings, the outcomes of various studies, and the areas still under debate.

\section{Video assisted thoracoscopic surgery (VATS)}

The literature is dominated by numerous studies and reports of using VATS for the treatment of recurrent pneumothorax and pneumothorax with persistent air leak, as well as first time presentations associated with tension or bilaterality. It has been advocated even as a routine for first time primary spontaneous pneumothorax, for the reason of cost effectiveness. ${ }^{23}$ Several studies have demonstrated benefits attributable to shorter hospital stay and reduced complication and recurrence rates compared with conservative management. VATS is certainly more valuable if the patient is unreliable for follow ups; or if the patient has a high risk occupation (for example, frequent air travelling and scuba diving). As the routine use of the technique is subject to availability, and as this article aims to consider the topic from the practical perspective of the emergency department physician, the development of VATS, even though acknowledged, will not be the main focus here.

\section{British Thoracic Society Guidelines ${ }^{1}$}

The British Thoracic Society (BTS) guidelines for the management of spontaneous pneumothorax were drawn up in 1993 after consultation with over 150 British respiratory physicians and thoracic surgeons. The key direction of these guidelines in management was the more conservative approach of observation and the use of simple aspiration with outpatient follow up. AC Miller discusses five main principles and concepts related to the derivation of the guidelines ${ }^{4}$; and these views are summarised below:

1 The presence of intrapleural air is not in itself necessarily an indication for intervention.

2 Management should depend more on the clinical symptoms than the size of the pneumothorax. Younger patients tolerate pneumothorax much better than older patients with chronic lung disease and diminished respiratory reserves. A young and fit patient with a large pneumothorax without significant symptoms (dyspnoea) can be observed as outpatient.

3 Complete collapse can be treated effectively by simple aspiration.

4 Tension pneumothorax developing from primary spontaneous pneumothorax is extremely rare. It occurs mainly with secondary pneumothorax and pneumothorax caused by positive pressure ventilation.

5 When drainage of air is required, simple aspiration should always be attempted first. This applies even to patients with chronic lung disease.

The technique of simple aspiration is described in the BTS guidelines. It involves the 
use of a 16 gauge cannula under local anaesthetic at the second intercostal space, mid-clavicular line, with a $50 \mathrm{ml}$ syringe and a three way tap. Aspiration is discontinued if more than 2.5 litres are aspirated.

Offering major scientific support for the BTS guidelines was a study by Harvey and Prescott, ${ }^{5}$ comparing simple aspiration with intercostal drainage by tube thoracostomy. Seventy three patients from 13 hospitals who presented with a spontaneous pneumothorax requiring a drainage procedure were randomly allocated to be treated with either simple aspiration or intercostal drainage. The conclusions of this study are that simple aspiration is less painful, the hospital stay is shorter, and there is no difference in recurrence rate after one year, compared with intercostal drainage. It is also noted that simple aspiration is associated with less likelihood of the subsequent requirement of pleurectomy.

Since 1993, the BTS guidelines are thought to "have become standard throughout the UK" and have been "included in emergency, general and respiratory textbooks". ${ }^{6}$ However, the move for this more conservative approach is not without its critics. Grant ${ }^{7}$ challenged the methodology of the study by Harvey and Prescott. ${ }^{5}$ The two groups (simple aspiration and intercostal drainage) studied were ill matched. There was a higher frequency of more severe cases with complete lung collapse in the intercostal drainage group. According to Grant $^{7}$ this could explain at least the observation that intercostal drainage was associated with an increased need for subsequent pleurectomy.

Baumann and Strange also disagreed with the use of observation and of simple aspiration. ${ }^{8}$ They had concerns about the safety of outpatient observation. They felt that intercostal drainage would allow air leak monitoring and could provide recurrence prevention by pleurodesis if necessary. They were in favour of a more aggressive approach, and recurrence prevention would be considered for the first presentation of a spontaneous pneumothorax. ${ }^{9}$ They commented that the UK guidelines were only supported by four references, and that there was no documentation of the degree of conflicting opinion among the specialists consulted before the drawing up of the guidelines. There is a lack of objective data describing the current UK experience of adherence to the guidelines. A truly evidencebased international guideline has yet to be evolved.

\section{Audit in the management of spontaneous pneumothorax}

There are two recent studies in the literature investigating the outcomes of management of spontaneous pneumothorax, as well as the degree of compliance to the BTS guidelines, since these were implemented.

Soulsby ${ }^{10}$ retrospectively studied the case notes of all patients with spontaneous pneumothorax who attended the accident and emergency (A\&E) department of a large district general hospital in England, over a four year period. One hundred and fifteen cases were identified. The findings of note were:

1 Forty three pneumothoraces were aspirated, of which $23(58 \%)$ were successful; 8 $(18.6 \%)$ with little or no improvement; and $12(27.9 \%)$ were successful initially but developed increasing size of pneumothorax within 72 hours.

2 The factors significantly associated with failure of aspiration were age over 50 , chronic lung disease and aspiration exceeding 2.5 litres $(\mathrm{p}<0.01)$.

3 The failure rate of aspiration for patients without chronic lung disease was $28.6 \%$, which is better than the overall failure rate (which includes aspiration of those with chronic lung disease).

4 All patients admitted for observation without chronic lung disease $(n=16)$ eventually had no intervention and could have been safely sent home.

5 Overall only $20.8 \%$ of episodes were treated correctly by BTS guidelines. This finding is in contrast with Miller's statement quoted above, ${ }^{6}$ that the BTS guidelines have been widely practised as standard protocol.

An audit of the management of spontaneous pneumothorax, performed by Courtney and McKane ${ }^{11}$ of Northern Ireland also suggested that clinical practice differed from guidelines issued by the British Thoracic Society. In his audit, simple aspiration was attempted in only seven of 65 patients.

The effectiveness of simple aspiration was evaluated by $\mathrm{Ng}$, Chan and Lee ${ }^{12}$ in Singapore in a study involving 34 patients whose spontaneous pneumothorax would otherwise have been treated by intercostal tube drainage. The procedure was successful in 25 patients (26.5\% failure rate) and patients' symptoms were relieved. Factors noted to be associated with success were:

1 Age below 50 .

2 No underlying lung disease.

3 Pneumothorax size not more than 50\%.

4 Volume of air aspirated less than $3000 \mathrm{ml}$.

5 No previous pneumothorax.

However, the above conclusions were drawn from figures that, although impressive, were not accompanied by the corresponding statistical analyses for significance.

The success rate of simple needle aspiration for spontaneous pneumothorax, reported by Andrivet et $a l^{13}$ in France was $68.5 \%$. These workers pointed to the risk of relapse several hours after an apparent success, and although none of their failed cases was complicated by tension pneumothorax, they questioned the safety of outpatient management of spontaneous pneumothorax by simple aspiration. The BTS guidelines ${ }^{1}$ however do advocate discharge (without observation) with follow up after successful simple aspiration of spontaneous pneumothorax without chronic lung disease.

Campisi and Voitk ${ }^{14}$ studied the appropriateness and potential cost-savings of routine outpatient management of spontaneous pneumothorax using closed tube thoracostomy and the Heimlich valve. Fourteen consecutive cases 


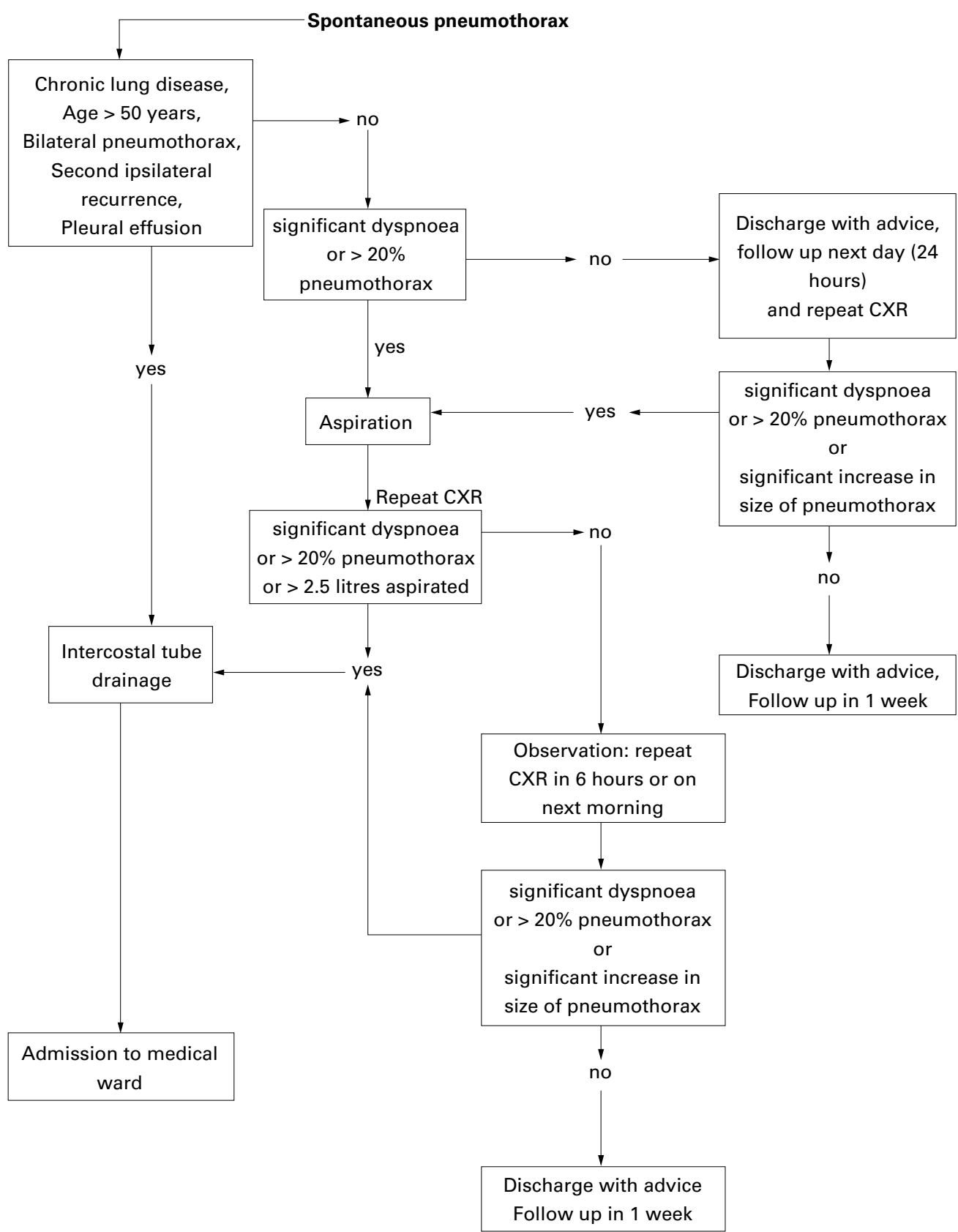

Figure 1 Suggested guidelines for the initial management of spontaneous pneumothorax.

were treated as outpatients by one surgeon in a community hospital setting in Canada. "All 14 patients were successfully managed although 3 required overnight admission due to anxiety, pain or vasovagal reaction.”

In the United States currently, the approach of emergency physicians to the management of spontaneous pneumothorax differs somewhat from that recommended by the British Thoracic Society guidelines. This is reflected by reference to major authoritative US textbooks in emergency medicine. ${ }^{15-19}$ Most still teach that pre-existing chronic lung disease is a definite indication for intercostal tube drainage. Most will aspirate a large ( $>20 \%$ ) pneumothorax even in the absence of significant dyspnoea.

McEwen ${ }^{15}$ suggests conservative treatment for a small pneumothorax $(<15 \%)$ if the patient is relatively asymptomatic and other- wise reliable and fit. One hundred per cent oxygen may be administered to hasten absorption of intrapleural air. The patient is observed in the emergency department for six hours. If repeat chest radiography at that time shows no increase in pneumothorax size, the patient may be discharged with appropriate advice, and reviewed again in 24 hours for a repeat radiography. The patient should be followed up weekly with progress radiographs until full resolution occurs.

Simple aspiration is performed to reduce a large or moderate pneumothorax $(>20 \%)$ to a small one (irrespective of the presence or absence of symptoms in the patient). If the patient has significant lung disease or concurrent medical problems, haemothorax, pleural effusion and bilateral pneumothorax, then simple aspiration is contraindicated and intercos- 
tal tube drainage should be performed instead. After aspiration, chest radiography is performed and significant residual pneumothorax can be reaspirated once. The patient is discharged if there is no significant dyspnoea after six hours of observation and a repeat radiograph at that time shows no recurrence of pneumothorax. The patient is to return immediately if significant dyspnoea occurs, and to return in 24 hours for follow up and further repeat chest radiography.

Simple aspiration can also be performed by using commercial ready to use aspiration kits. The plastic catheter can be secured to the chest wall after aspiration, and repeat aspiration performed if required, through the same catheter. It can also be attached to a Heimlich valve if pneumothorax recurs or persists.

In Australia, a leading up to date $A \& E$ handbook ${ }^{20}$ still advocates that intercostal tube drainage (instead of simple aspiration) is indicated if the patient has pre-existing chronic lung disease. Even though the book refers its readers to the BTS guidelines on this subject, the practice taught is a notable departure from the BTS recommendation that simple aspiration and observation be the management for patients even with chronic lung disease.

The size of pneumothorax can be predicted radiologically by measuring the average intrapleural distance. ${ }^{12}{ }^{1521}$ In a study of 11 patients with pneumothoraces ranging in size from $16 \%$ to $100 \%$, the mean rate of re-expansion was $1.8 \%$ per day. ${ }^{22}$ Traditional opinion is that expiratory films are better at detecting subtle pneumothoraces. Recently however, it has been suggested that the routine addition of expiratory chest films does not improve the diagnostic yield in patients suspected to have pneumothorax. ${ }^{23}$

\section{Conclusion}

There are several possible methods for the initial treatment of spontaneous pneumothorax. Practices today still vary widely from centre to centre, and range from observation with simple aspiration to the more aggressive approach with various methods of recurrence prevention (including VATS) for all first time spontaneous pneumothoraces. An approach somewhere in the middle of these extremes is probably the most appropriate. The advantages of the use of simple aspiration instead of intercostal tube drainage are that, if successful, needle aspiration means less pain, less scarring, and shorter hospital stay for the patient. However, with the success rate of simple aspiration ranging from only $58 \%$ to $80 \%,{ }^{510} 12$ the consequence of failed aspiration also becomes quite significant. For the patient who has had an unsuccessful aspiration, and eventual resort to intercostal tube drainage, this may mean added anxiety and pain from an extra procedure of aspiration, the frustration of failure after a period of observation, the stress of having to return to hospital with a deterioration of condition, and an inevitably longer hospital stay or period of morbidity compared with what would otherwise have happened if a drain were inserted right in the first instance. The safest and most cost effective treatment protocol for a particular centre should be used. There is definitely a need for more good quality research in this area.

Suggested guidelines by the author, who practises in the $\mathrm{A} \& \mathrm{E}$ department of a major university teaching hospital in Hong Kong, are included here for reference (fig 1). This local preference is based upon best available scientific evidence and a thorough and balanced review of the current opinions of internationally respected authorities, as quoted, described and discussed above. Based on the reported failure rates of simple aspiration, and the finding that failure is associated with the following factors: age above 50 years, chronic lung disease, recurrent pneumothorax, aspiration of more than 2.5 litres, it is recommended that intercostal tube drainage should be performed in the presence of any of these factors.

\section{Contributors}

Stewart S W Chan, Senior Medical Officer/Adjunct Assistant Professor, Department of Accident and Emergency, Prince of Professor, Department of Accident and Emergency, Prince of sor Robert Cocks, Director, Accident and Emergency Medicine Academic Unit, The Chinese University of Hong Kong (guarantor). Dr Wai Lun Cheung, Consultant and Chief of (guarantor). Dr Wai Lun Cheung, Consultant and Chief of
Service/Associate Professor, Department of Accident and Service/Associate Professor, Department of Accident and
Emergency, Prince of Wales Hospital, The Chinese University Emergency, Prince of Wales Hospital, The Chinese University
of Hong Kong (guarantor). Stewart Chan initiated the research, reviewed literature, discussed core ideas and wrote the paper. Robert Cocks gave valuable comments and edited the paper. Cheung Wai Lun initiated the research, discussed core ideas and gave valuable comments.

Funding: none.

Conflicts of interest: none.

1 Miller AC, Harvey JE on behalf of Standards of Care Committee, British Thoracic Society. Guidelines for the management of spontaneous pneumothorax. BMF 1993;307:114-16.

2 Schramel FM, Posthumus PE, Vanderschueren RG. Current aspects of spontaneous pneumothorax. Eur Respir $\mathcal{f}$ 1997;10:1372-9.

3 Schramel FM, Sutedja TG, Broker JC, et al. Costeffectiveness of video-assisted thoracoscopic surgery vs conservative treatment for first time or recurrent spontaneconservative treatment for first time or recurrent

4 Miller AC. Management of spontaneous pneumothorax: Miller AC. Management of spontaneous pneum
back to the future. Eur Respir $\mathcal{7} 1996 ; 9: 1773-4$.

5 Harvey J, Prescott RJ. Simple aspiration versus intercostal tube drainage for spontaneous pneumothorax in patients with healthy lungs. BMF 1994;309:1338-9.

6 Miller AC. Treatment of spontaneous pneumothorax: the clicicians's perspective on pneumothorax management [letter]. Chest 1998;113:1423-5.

7 Grant IW. Simple aspiration for spontaneous pneumothorax [letter; comment]. BMF 1995;310:468-9.

8 Baumann $\mathrm{MH}$, Strange C.[Communications to editor] Chest 1998;113:1424-5.

9 Baumann MH, Strange C. Treatment of spontaneous pneumothorax: a more aggressive approach? Chest 1997; 112:789-804.

10 Soulsby T. British Thoracic Society guidelines for the management of spontaneous pneumothorax: do we comply with them and do they work? f Accid Emerg Med 1998;15: with the

11 Courtney PA, McKane WR. Audit of the management of

spontaneous pneumothorax. Ulster Med f 1998;67:41-3.
Ng AW, Chan KW, Lee SK. Simple aspiration of pneumot$\mathrm{Ng}$ AW, Chan KW, Lee SK. Simple aspit
horax. Singapore Med f 1994;35:50-2.

13 Andrivet P, Djedaini K, Teboul JL, et al. Spontaneous pneumothorax. Comparison of thoracic drainage vs immediate or delayed needle aspiration. Chest 1995;108:335-9.

14 Campisi P, Voitk AJ. Outpatient treatment of spontaneous pneumothorax in a community hospital using a Heimlich flutter valve: a case series. F Emerg Med 1997;15:115-19.

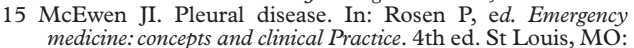
Mosby-Year Books, 1998:1518-20.

16 Wisdom K. Spontaneous and iatrogenic Pneumonthorax. In: Tintinalli JE, Ruiz E, Krome RL, eds. Emergency medicine. A comprehensive guide. 4th ed. New York: McGrawcine. A comprehens

17 Hauda II WE. Tube and needle thoracostomy. In: Howell JM, ed. Emergency medicine. Philadelphia: WB Saunders, 
18 Howell JM. Disorders of the pleura and mediastinum. In: Howell JM, ed. Emergency medicine. Philadelphia:WB

19 Dunmire SM. Spontaneous pneumothorax and pneumomediastinum. In: Harwood-Nuss AL, ed. The clinical practice of emergency medicine. 2nd ed. Philadelphia: LippincottRaven, 1996:663-4.

20 Brown AFT. Accident and emergency diagnosis and management. 3rd ed. London: Butterworth Heinemann, 1996 $23-4$.
21 Rhea JT, Deluca SA, Greene RE. Determining the size of pneumothorax in the upright patient. Radiology 1982;144: pneum $733-6$.

22 Flint K, Hillawi AH, Johnson AM. Conservative management of spontaneous pneumothorax. Lancet 1984;i:6878.

23 Schramel FM, Golding RP, Haakman CD, et al. Expiratory chest X-rays do not improve visibility of small apical pneumothoraces by enhanced contrast. Eur Respir f 1996;9: $406-9$. 